\title{
THE WELL-POSEDNESS OF A SWIMMING MODEL IN THE 3-D INCOMPRESSIBLE FLUID GOVERNED BY THE NONSTATIONARY STOKES EQUATION
}

\author{
ALEXANDER KHAPALOV \\ Department of Mathematics \\ Washington State University, Pullman, WA 99164-3113, USA \\ e-mail: khapala@math.wsu.edu
}

\begin{abstract}
We introduce and investigate the well-posedness of a model describing the self-propelled motion of a small abstract swimmer in the 3-D incompressible fluid governed by the nonstationary Stokes equation, typically associated with low Reynolds numbers. It is assumed that the swimmer's body consists of finitely many subsequently connected parts, identified with the fluid they occupy, linked by rotational and elastic Hooke forces. Models like this are of interest in biological and engineering applications dealing with the study and design of propulsion systems in fluids.
\end{abstract}

Keywords: swimming models, coupled PDE/ODE systems, nonstationary Stokes equation.

\section{Introduction and 3-D model setting}

It seems that the first quantitative research in the area of the swimming phenomenon was aimed at the biomechanics of specific biological species (e.g., Gray,1932; Gray and Hancock, 1955; Taylor, 1951; 1952; Wu, 1971; Lighthill, 1975). These efforts resulted in the derivation of a number of mathematical models (linked by the size of the Reynolds number) for swimming motion in the whole $\mathbb{R}^{2}$ - or $\mathbb{R}^{3}$-spaces with the swimmer to be used as the reference frame (see, e.g., the work of Childress (1981) and the references therein). Such an approach, however, requires some modification if one wants to track the actual position of a swimmer in a fluid.

It should be noted that the classical mathematical issue of the well-posedness of a swimming model as a system of PDEs was addressed for the first time by Galdi (1999) (see also Galdi, 2002) for a model of swimming micromotions in $\mathbb{R}^{3}$ in the fluid governed by the stationary Stokes equation with the swimmer serving as the reference frame.

A different modeling approach was proposed by Peskin in computational mathematical biology (see the works of Peskin (1977), Fauci and Peskin (1988), Fauci (1993), Peskin and McQueen (1994), Tytell et al. (2010) and the references therein), where a swimmer was modeled as an immaterial immersed boundary identified with the fluid, further discretized for computational purposes on some grid. In this case a fluid equation is to be complemented by a coupled infinite dimensional differential equation for the aforementioned "immersed boundary".

In this paper we deal with the swimming phenomenon in the framework of non-stationary PDEs along the immersed body approach summarized by Khapalov (2010), who was also inspired by the ideas of the above-cited Peskin's method, introduced a 2-D model for "small" flexible swimmers assuming that their bodies are identified with the fluid occupying their shapes (Khapalov, 2005). This approach views such a swimmer as an already discretized, aforementioned immersed boundary supported on the respective grid cells (see, e.g., Figs. 1 and 2). Our model offered two novel features: (a) it was set in $A$ bounded domain with (b) the governing equations to be a fluid equation coupled with a system of ODEs describing the spatial position of the swimmer within the space domain. We established the well-posedness of this model up to the contact either between the swimmer's body parts or with the boundary of the space domain. The need for such a type of models was motivated by the intention to investigate controllability properties of swimming phenomenon (see Khapalov, 2010). Our goal in this paper is to introduce a possible 3-D extension of this model and to investigate its well-posedness. 
1.1. Motivation: Work for the 2-D swimming model and its implications for the 3-D case. In our previous work (Khapalov, 2010) we studied the model of a swimmer in a 2-D fluid governed by the non-stationary Stokes equation of Fig. 1.

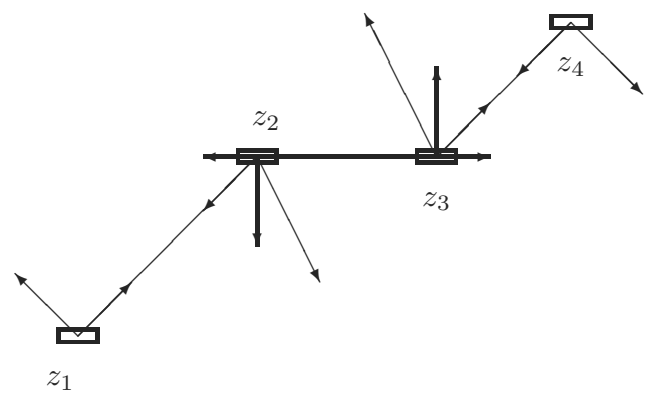

Fig. 1. Swimmer composed of four body parts.

The body of the swimmer was modeled as a collection of $n$ open bounded sets of non-zero measure (in Fig. 1 they are parallelograms), identified with the fluid within the space they occupy. We assumed that their centers of mass $z_{i}(t)$ 's are subsequently linked by two types of internal swimmer forces (i.e., their sum is zero) shown in Fig. 1:

- The co-linear pairs of elastic forces, acting in the opposite directions, to connect any two of the adjacent points $z_{i}(t)$ 's. They are to preserve the spatial structure of the swimmer at hand.

- The pairs of rotational forces, acting about any of the "mid" points $z_{i}(t)$ 's, to make the adjacent points $z_{i-1}(t)$ and $z_{i+1}(t)$ rotate towards each other about $z_{i}(t)$ perpendicular to the lines connecting them to $z_{i}(t)$. To satisfy the 3rd Newton Law, the pairs of the rotational forces are complemented by the matching counter-forces applied to $z_{i}(t)$ 's.

In the work of Khapalov (2010, Chapters 10-15) (we refer to the bibliography therein for the separately published papers), the following results were presented for the above 2-D swimming model:

1. We discussed how the geometric shape of an object (not necessarily a swimmer) affects the forces acting upon it when it is placed inside the fluid. This phenomenon is directly responsible for the fact of principal possibility of swimming, i.e., self-propulsion (Chapter 13)

2. The well-posedness of the aforementioned 2-D swimming model was analyzed (Chapter 12) (see also Khapalov and Eubanks, 2009).

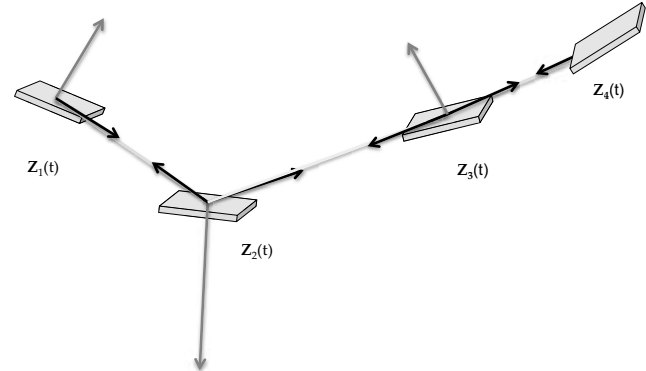

Fig. 2. Example of a 4-parallelepiped swimmer with all elastic forces active and with rotational forces about $z_{2}(t)$ only.

3. We discussed its local controllability (Chapter 14).

4. The global controllability of the same model was also studied (Chapter 15).

In this paper we attempt to follow the same research plan but now in the 3-D case, which, while retaining many similarities with the 2-D case, is substantially more challenging in many aspects of the above research plan due to the differences between the 2-D and 3-D geometries.

In our previous work (Khapalov and Trinh, 2013) we already obtained new geometric results for the forces acting on objects in the 3-D fluid, governed either by the non-stationary Stokes equation or by the Navier-Stokes equation (see point 1 above), with the emphasis on the parallelepipeds of different proportions and the balls.

In this paper we focus on the introduction of a 3-D swimming model as a possible extension of the above-mentioned 2-D model and its well-posedness (compare to point 2 above). An example of a 3-D model of our interest is shown in Fig. 2

1.2. Rotational forces in 3-D. In the two-dimensional case the rotational forces can be described by making use of the matrix

$$
A=\left(\begin{array}{cc}
0 & 1 \\
-1 & 0
\end{array}\right)
$$

rotating each vector at hand by $90^{\circ}$. In particular, we can describe the rotational forces, induced by the point $z_{2}$ in Fig. 1 at the adjacent points $z_{1}$ and $z_{3}$, as $v_{1}(t) A\left(z_{1}(t)-\right.$ $\left.z_{2}(t)\right)$ and $-v_{1}(t) A\left(z_{3}(t)-z_{2}(t)\right)$, respectively. The scalar parameter $v_{1}(t)$ is introduced to allow one to chose the direction of rotation between folding or unfolding motions, as well as to assign the value of the magnitude of the rotational forces.

Unlike in the 2-D case when all rotations lie in the same plane and with only two options to choose between 
the clockwise and counterclockwise rotations, in three dimensions each vector can rotate by $90^{\circ}$ in infinitely many directions and planes. Their description by means of a single matrix is no longer an option. Therefore, in our further 3-D description of rotational forces, we join them in triplets to identify the plane in which these forces will act along with the respective countering forces (as shown in Fig. 2). This will also allow of the 3rd Newton Law to hold for each triplet.

To this end, assuming that the body of the swimmer at hand consists of $n$ parts with centers of mass at $z_{i}(t)$ 's, for any triplet $z_{i-1}(t), z_{i}(t), z_{i+1}(t), i=2, \ldots, n-1$ we introduce a pair of nonlinear mappings $\left\{A_{i}, B_{i}\right\}$, acting respectively on the vectors $z_{i-1}(t)-z_{i}(t)$ and $z_{i+1}(t)-$ $z_{i}(t)$, so that

(i) we have

$$
\begin{aligned}
& A_{i}\left(z_{i-1}(t)-z_{i}(t)\right), B_{i}\left(z_{i+1}(t)-z_{i}(t)\right) \\
\in & \operatorname{span}\left\{z_{i-1}(t)-z_{i}(t), z_{i+1}(t)-z_{i}(t)\right\} \subset \mathbb{R}^{3},
\end{aligned}
$$

in the case when $z_{i-1}(t), z_{i}(t), z_{i+1}(t)$ do not form $a$ straight line;

(ii) if otherwise, the respective images $A_{i}\left(z_{i-1}(t)-\right.$ $\left.z_{i}(t)\right)$ and $B_{i}\left(z_{i+1}(t)-z_{i}(t)\right)$ must lie in any plane (the same for both images) chosen among infinitely many of those which also contain $z_{i-1}(t), z_{i}(t), z_{i+1}(t)$;

(We also assume that, for $i=2, \ldots, n-1$.)

(iii) we have

$$
\begin{aligned}
& \left(z_{i-1}(t)-z_{i}(t)\right)^{\prime}\left[A_{i}\left(z_{i-1}(t)-z_{i}(t)\right)\right]=0, \\
& \left(z_{i+1}(t)-z_{i}(t)\right)^{\prime}\left[B_{i}\left(z_{i+1}(t)-z_{i}(t)\right)\right]=0,
\end{aligned}
$$

where prime denotes the transposition in $\mathbb{R}^{3}$;

(iv) we have

$$
\begin{aligned}
& \left\|A_{i}\left(z_{i-1}(t)-z_{i}(t)\right)\right\|_{\mathbb{R}^{3}}=\left\|z_{i-1}(t)-z_{i}(t)\right\|_{\mathbb{R}^{3}}, \\
& \left\|B_{i}\left(z_{i+1}(t)-z_{i}(t)\right)\right\|_{\mathbb{R}^{3}}=\left\|z_{i+1}(t)-z_{i}(t)\right\|_{\mathbb{R}^{3}} ;
\end{aligned}
$$

(v) and the directions of vectors $A_{i}\left(z_{i-1}(t)-z_{i}(t)\right)$ and $-B_{i}\left(z_{i+1}(t)-z_{i}(t)\right)$ are such that they correspond to either a folding or an unfolding motion of the respective vectors $z_{i-1}(t)-z_{i}(t)$ and $z_{i+1}(t)-$ $z_{i}(t)$ towards each other about the point $z_{i}(t)$, as illustrated in Fig. 2.

Example 1. When the points $z_{i-1}(t), z_{i}(t), z_{i+1}(t)$ do not form a straight line, the above assumptions can be satisfied along the following strategy to chose $\left\{A_{i}, B_{i}\right\}$ 's, making use of the cross-products:

$$
\left.A_{i}\left(z_{i-1}(t)-z_{i}(t)\right)=e_{1}(t) \| z_{i-1}(t)-z_{i}(t)\right) \|_{\mathbb{R}^{3}},
$$

$$
\begin{aligned}
B_{i}\left(z_{i+1}(t)\right. & \left.\left.-z_{i}(t)\right)=e_{2}(t) \| z_{i+1}(t)-z_{i}(t)\right) \|_{\mathbb{R}^{3}}, \\
& e_{i}(t)=\frac{v_{i}(t)}{\left\|v_{i}(t)\right\|_{\mathbb{R}^{3}}}, \quad i=1,2, \\
v_{1}(t)= & \left(z_{i-1}(t)-z_{i}(t)\right) \times\left[\left(z_{i-1}(t)-z_{i}(t)\right)\right. \\
& \left.\times\left(z_{i+1}(t)-z_{i}(t)\right)\right], \\
v_{2}(t)= & {\left[\left(z_{i+1}(t)-z_{i}(t)\right) \times\left(z_{i+1}(t)-z_{i}(t)\right)\right] } \\
& \times\left(z_{i+1}(t)-z_{i}(t)\right) .
\end{aligned}
$$

In this example $\left\{A_{i}, B_{i}\right\}, i=2, \ldots, n-1$ are defined by the spatial positions of the points $z_{i-1}(t), z_{i}(t), z_{i+1}(t)$ only.

Alternative methods to construct $\left\{A_{i}, B_{i}\right\}$ 's can, for example, be (i) the use of the technique of the Gramm-Schmidt orthogonalization procedure for vectors $\left.\left\{z_{i-1}(t)-z_{i}(t), z_{i+1}(t)-z_{i}(t)\right)\right\}$, or (ii) the use of rotational $[3 \times 3]$ matrices similar to the $[2 \times 2]$ matrix $A$, mentioned in the beginning of this subsection for the 2-D case. Such matrices, however, are to be constructed individually for each of the planes spanned by the aforementioned two vectors at each different moment of time.

Steering options in 3-D. In the case when the points $z_{i-1}(t), z_{i}(t), z_{i+1}(t)$ do form a straight line, i.e., they do not define a unique plane, the cross-product approach of Example 1 will not work. Instead, at the respective moments of time (we will further call them the "plane choice instants") we will have infinitely many options to assign the plane for the rotational forces about the point $z_{i}(t)$ among those that also contain the points $z_{i-1}(t), z_{i}(t), z_{i+1}(t)$. In terms of applications, in this situation one has an option to make a decision on how to select $\left\{A_{i}, B_{i}\right\}$ 's in a "concrete manner", i.e., as opposed to a "universal preassigned" formula representation (as in Example 1 or similar). Such a decision making process can be viewed as one of the critical elements of controlling actions over the motion of the swimming device at hand, intrinsic to the 3-D space.

Discontinuity of $\left\{\boldsymbol{A}_{\boldsymbol{i}}, \boldsymbol{B}_{\boldsymbol{i}}\right\}$. We can view each pair $\left\{A_{i}, B_{i}\right\}$ as a mapping (denote it by $\boldsymbol{\Pi}_{i}$ ), defined as in the above on any pair of vectors $\{\mathbf{a}, \mathbf{b}\}$ in place of $\left\{z_{i-1}(t)-z_{i}(t), z_{i+1}(t)-z_{i}(t)\right\}$ :

$$
\begin{gathered}
\boldsymbol{\Pi}_{i}=\left\{A_{i}, B_{i}\right\}: \\
\left(\mathbb{R}^{3}\right)^{2} \ni\{\mathbf{a}, \mathbf{b}\} \rightarrow\left\{A_{i} \mathbf{a}, B_{i} \mathbf{b}\right\} \in\left(\mathbb{R}^{3}\right)^{2} .
\end{gathered}
$$

Any such mapping is discontinuous at any point $\left\{\mathbf{a}_{o}, \mathbf{b}_{o}\right\}$, if $\mathbf{a}_{o}$ and $\mathbf{b}_{o}$ are co-linear. Indeed, on the one hand, we have an infinite choice of planes to assign for the images $\left\{A_{i} \mathbf{a}, B_{i} \mathbf{b}\right\}$ when $\{\mathbf{a}, \mathbf{b}\}$ are co-linear, but, 
regardless of this choice, a single pre-determined plane for them, otherwise. This means that the image of any neighborhood of the aforementioned co-linear pair $\left\{\mathbf{a}_{o}, \mathbf{b}_{o}\right\}$ in $\left(\mathbb{R}^{3}\right)^{2}$ due to $\boldsymbol{\Pi}_{i}$ will have pairs of vectors lying in any plane in $\mathbb{R}^{3}$. It remains to recall that $\left\{A_{i}, B_{i}\right\}$ 's preserve the magnitudes of vectors they act upon, while making them perpendicular to the original vectors. This discontinuity property does not occur in the 2-D case.

Our main result below, Theorem 1, deals with the well-posedness of the model at hand for the time-intervals between the aforementioned "plane choice instants" (see also Remark 2, parts 1 and 3 below).

1.3. Problem formulation for 3-D swimming model. In this paper we study the following mathematical model associated with the one shown in Fig. 2. It consists of two coupled systems of equations: a PDE system-for the fluid, governed by the nonstationary 3-D Stokes equation, and an ODE system-for the position of the swimmer in it:

$$
\frac{\partial y}{\partial t}=\nu \Delta y+F(z, v)-\nabla p \quad \text { in } \quad Q_{T}=\Omega \times(0, T),
$$

$$
\operatorname{div} y=0 \text { in } \quad Q_{T}, \quad y=0 \quad \text { in } \Sigma_{T}=\partial \Omega \times(0, T) \text {, }
$$

$$
y=\left(y_{1}, y_{2}, y_{3}\right),\left.\quad y\right|_{t=0}=y_{0} \text { in } \Omega
$$

$$
\frac{\mathrm{d} z_{i}}{\mathrm{~d} t}=\frac{1}{\operatorname{mes}\left\{S_{i}(0)\right\}} \int_{S_{i}\left(z_{i}(t)\right)} y(x, t) \mathrm{d} x, \quad z_{i}(0)=z_{i 0},
$$

$i=1, \ldots, n, \quad n>2$, where for $t \in[0, T]:$

$$
\begin{aligned}
z(t)= & \left(z_{1}(t), \ldots, z_{n}(t)\right), \\
& z_{i}(t) \in \mathbb{R}^{3}, \quad i=1, \ldots, n \\
v(t)= & \left(v_{1}(t), \ldots, v_{n-2}(t)\right) \in \mathbb{R}^{n-2}, \\
F(z, v) & \\
= & \sum_{i=2}^{n}\left[\xi_{i-1}(x, t) k_{i-1}\right. \\
& \times \frac{\left(\left\|z_{i}(t)-z_{i-1}(t)\right\|_{\left.\mathbb{R}^{3}-l_{i-1}\right)}\left(z_{i}(t)-z_{i-1}(t)\right)\right.}{\left\|z_{i}(t)-z_{i-1}(t)\right\|_{\mathbb{R}^{3}}} \\
& +\xi_{i}(x, t) k_{i-1} \frac{\left(\left\|z_{i}(t)-z_{i-1}(t)\right\|_{\mathbb{R}^{3}}-l_{i-1}\right)}{\left\|z_{i}(t)-z_{i-1}(t)\right\|_{\mathbb{R}^{3}}} \\
& \left.\times\left(z_{i-1}(t)-z_{i}(t)\right)\right] \\
& +\sum_{i=2}^{n-1} v_{i-1}(t)\left\{\xi_{i-1}(x, t)\left(A_{i}\left(z_{i-1}(t)-z_{i}(t)\right)\right)\right. \\
& -\xi_{i+1}(x, t) \frac{\left\|z_{i-1}(t)-z_{i}(t)\right\|_{\mathbb{R}^{3}}^{2}}{\left\|z_{i+1}(t)-z_{i}(t)\right\|_{\mathbb{R}^{3}}^{2}} \\
& \left.\times\left(B_{i}\left(z_{i+1}(t)-z_{i}(t)\right)\right)\right\}
\end{aligned}
$$

$$
\begin{aligned}
& -\sum_{i=2}^{n-1} \xi_{i}(x, t) v_{i-1}(t)\left\{\left(A_{i}\left(z_{i-1}(t)-z_{i}(t)\right)\right)\right. \\
& \left.-\frac{\left\|z_{i-1}(t)-z_{i}(t)\right\|_{\mathbb{R}^{3}}^{2}}{\left\|z_{i+1}(t)-z_{i}(t)\right\|_{\mathbb{R}^{3}}^{2}}\left(B_{i}\left(z_{i+1}(t)-z_{i}(t)\right)\right)\right\} .
\end{aligned}
$$

In the above, $\Omega$ is a bounded domain in $\mathbb{R}^{3}$ with boundary $\partial \Omega$ of class $C^{2}, y(x, t)$ and $p(x, t)$ are respectively the velocity and the pressure of the fluid at point $x=\left(x_{1}, x_{2}, x_{3}\right) \in \Omega$ at time $t$, while $\nu$ is a kinematic viscosity constant. Let us explain the terms in (1)-(3) in detail (see also Section 1.1 for the 2-D case).

The swimmer in (1)-(3) is modeled as a collection of $n$ bounded sets $S_{i}\left(z_{i}(t)\right), i=1, \ldots, n$, of non-zero measure (such as balls, parallelepipeds, etc.), identified with the fluid within the space they occupy. These sets are assumed to be open bounded connected sets with the points $z_{i}(t)$ 's to be their centers of mass. The sets $S_{i}\left(z_{i}(t)\right)$ are viewed as given sets $S_{i}(0)$ ("0" stands for the origin) that have been shifted to the respective positions $z_{i}(t)$ without changing their orientation in space. Respectively, for $i=1, \ldots, n$,

$$
\xi_{i}(x, t)= \begin{cases}1, & \text { if } x \in S_{i}\left(z_{i}(t)\right), \\ 0, & \text { if } x \in \Omega \backslash S_{i}\left(z_{i}(t)\right) .\end{cases}
$$

Throughout the paper, we assume that each $S_{i}(0)$ lies in a "small" neighborhood of the origin of a given radius $r>$ 0 , while $S_{i}(a)$ denotes the set $S_{i}(0)$ shifted to point $a$. Write

$$
\begin{aligned}
S^{0} & =\max _{i=1, \ldots, n}\left\{\operatorname{mes}\left\{S_{i}(0)\right\}\right\}, \\
S_{0} & =\min _{i=1, \ldots, n}\left\{\operatorname{mes}\left\{S_{i}(0)\right\}\right\} .
\end{aligned}
$$

We assume that these sets are subsequently linked by the internal elastic structural and rotational forces as described in Sections 1.1-1.2. Their sum is zero. We assume that a force applied to a point $z_{i}(t)$ acts evenly over the set $S_{i}\left(z_{i}(t)\right)$, and, as such, it creates an external force on the fluid surrounding $S_{i}\left(z_{i}(t)\right)$

The elastic forces act according to Hooke's law when the distances between any two adjacent points $z_{i-1}(t)$ and $z_{i}(t), i=2, \ldots, n$, deviate from the respective given values $l_{i-1}>0, i=2, \ldots, n$ as described in the first sum in (3). The parameters $k_{i}>0, i=1, \ldots, n-1$, characterize the rigidity of the links $z_{i-1}(t) z_{i}(t), i=$ $2, \ldots, n$.

The second sum in (3) describes the rotational forces about any of the points $z_{i}(t), i=2, \ldots, n-1$, along with the matching counter-forces given in the third sum in (3). The magnitudes and directions of the rotational forces are determined by the given coefficients $v_{i}(t)$, $i=1, \ldots, n-2$. The choice of fractional coefficients at terms $A_{i}\left(z_{i+1}(t)-z_{i}(t)\right)$ in (3) ensures that the momentum of the swimmer's internal forces is conserved at any $t \in(0, T)$ (see calculations of Khapalov and 
Eubanks (2009) in the 2-D case, noting that the triplets $\left\{z_{i-1}(t), z_{i}(t), z_{i+1}(t)\right\}$ and respective rotational forces lie in one plane).

The movements of points $z_{i}(t), i=1, \ldots, n$, are determined by the average motions of the fluid within their respective supports $S_{i}\left(z_{i}(t)\right)$ as described in (2). These motions in turn define the motion of the center of mass of the swimmer, i.e., of the point $\frac{1}{n} \sum_{i=1}^{n} z_{i}(t)$.

Local and global approach to solutions of (1)-(3). Note that, when the adjacent points in the swimmer's body share the same position in space, the forcing term $F$ in (3) and hence the model (1)-(3) becomes undefined. While such a situation mathematically seems possible, it does not have to happen. First of all, one can address the issue of the local existence of solutions to (1)-(3) on some "small" time-interval $(0, T)$, assuming that initially the model (1)-(3) is well-defined in the above sense. This is the primary subject of this paper (see the next section). Then the question of global existence can be viewed as the issue of suitable selection of coefficients $v_{i}$ with the purpose to ensure that the aforementioned ill-posed situation is avoided.

In the model (1)-(3) we chose the fluid governed by the nonstationary Stokes equation which, along with its stationary version, is a typical choice of fluid for micro-swimmers (the case of low Reynolds numbers). The empiric reasoning behind this is that, due to the small size of swimmer, the inertia terms in the Navier-Stokes equation, containing the first-order derivatives in $t$ and $x$, can be omitted, provided that the frequency parameter of the swimmer at hand is a quantity of order unity. However, it was noted that a microswimmer (e.g., a nano-size robot) may use a rather high frequency of motion, which may justify at least in some cases the need for the term $y_{t}$ in the Stokes model equations. In general, it seems reasonable to suggest that the presence of this term (in a number of cases) can provide a better approximation of the Navier-Stokes equation than the lack of it. We also point out that in the works of Fauci and Peskin (1988), Fauci (1993) as well as Peskin and McQueen (1994) the full-size Navier-Stokes equation is used for micro-swimmers. It also seems that the methods we use for the nonstationary Stokes equation (as opposed to the stationary Stokes equation) may serve as a natural step toward swimming models, based on the Navier-Stokes equation.

Additional remarks on bibliography. We refer to the seminal work by Shapere and Wilczeck (1989) for an approach exploiting the idea that the swimmer's body shape transformations during the actual swimming process can be viewed as a set-valued map in time. The respective models describe the swimmer's position via such maps (see the works of Gurtin (1981), San Martin et al. (2008), Dal Maso et al. (2011) and the references therein). Some models treat these maps as prescribed a priori, in which case the crux of the problem is to identify which maps are admissible, i.e., compatible with the principle of self-propulsion of swimming locomotion. In the case when the aforementioned motion map is not prescribed a priori (i.e., it will be defined at each moment of time by the swimmer's internal forces and the interaction of its body with the resisting surrounding medium), the model will have to include extra equations (see, e.g., the work of Tytell et al. (2010) in the framework of the immersed boundary method and the references therein).

More recently, a number of significant efforts, both theoretical and experimental, have been made to study models of possible bio-mimetic mechanical devices which employ a change in their geometry, inflicted by internal forces, as the means of self-propulsion (see, e.g., the works of Hirose (1993), Mason and Burdick (2000), McIsaac and Ostrowski (2000), Martinez and Cortes (2001), Trintafyllou et al. (2000), Morgansen et al. (2001), Fukuda et al. (1995), Guo et al. (2002), Hawthorne et al. (2004) as well as Belter and Skrzypczyński (2010) and the references therein). It was also be recognized that the sophistication and complexity of the design of bio-mimetic robots give rise to control-theoretic methods (see, e.g., the works of Koiller et al. (1996), Khapalov (1999), McIsaac and Ostrowski (2000), Martinez and Cortes (2001), Trintafyllou et al. (2000), San Martin et al. (2007), Alouges et al. (2008) as well as Sigalotti and Vivalda (2009) and the references therein). It should be noted however, that the above-cited results deal with control problems in the framework of ODEs only.

A number of attempts have been made along these lines to introduce various reduction techniques to convert swimming model equations into systems of ODEs, namely, by making use of applicable analytical considerations, empiric observations and experimental data (see, e.g., the works of Becker et al. (2003), Kanso et al. (2005), San Martin et al. (2007) as well as Alouges et al. (2008) and the references therein).

\section{Main result: Local existence and uniqueness}

Let $\dot{J}(\Omega)$ denote the set of infinitely differentiable vector functions with values in $\mathbb{R}^{3}$ which have compact support in $\Omega$ and are divergence-free, i.e., $\operatorname{div} \phi=0$ in $\Omega$. Denote by $J_{o}(\Omega)$ the closure of this set in the $\left(L^{2}(\Omega)\right)^{3}$ norm and by $G(\Omega)$ the orthogonal complement of $J_{o}(\Omega)$ in $\left(L^{2}(\Omega)\right)^{3}$ (see, e.g., Ladyzhenskaya, 1963; Temam, 1984). In $\dot{J}(\Omega)$ introduce the scalar product (for $\phi_{1}(x)=$ 
$\left.\left(\phi_{11}, \phi_{12}, \phi_{13}\right), \phi_{2}(x)=\left(\phi_{21}, \phi_{22}, \phi_{23}\right)\right)$,

$$
\left[\phi_{1}, \phi_{2}\right]=\int_{\Omega} \sum_{j=1}^{3} \sum_{i=1}^{3} \phi_{1 j_{x_{i}}} \phi_{2 j_{x_{i}}} \mathrm{~d} x .
$$

Denote by $H(\Omega)$ the Hilbert space which is the completion of $\dot{J}(\Omega)$ in the norm

$$
\left\|\phi_{1}\right\|_{H(\Omega)}=\sqrt{\int_{\Omega} \sum_{j=1}^{3} \sum_{i=1}^{3} \phi_{1 j_{x_{i}}}^{2} \mathrm{~d} x} .
$$

Everywhere below we will assume the following.

Assumption 1. For a given $r>0$, defining the size of sets $S_{i}(0)$ in (4), assume that

$$
\begin{aligned}
& l_{i-1}>2 r, \quad i=2, \ldots, n ; \quad \overline{S_{i}}\left(z_{i}(0)\right) \subset \Omega, \\
& \left\|z_{i, 0}-z_{j, 0}\right\|_{\mathbb{R}^{3}}>2 r, \quad i, j=1, \ldots, n, i \neq j,
\end{aligned}
$$

and the sets $S_{i}(0), i=1, \ldots, n$, are such that

$$
\begin{gathered}
\int_{\left(S_{i}(0) \cup S_{i}(h)\right) \backslash\left(S_{i}(0) \cap S_{i}(h)\right)} \mathrm{d} x \\
=\int_{\Omega}\left|\xi_{i}(x)-\xi_{i}(x-h)\right| \mathrm{d} x \\
\leq C\|h\|_{\mathbb{R}^{3}} \quad \forall h \in B_{h_{0}}(0)
\end{gathered}
$$

for some positive constants $h_{0}$ and $C$, where $\xi_{i}(x)$ is the characteristic function of $S_{i}(0)$ and

$$
B_{h_{0}}(0)=\left\{x \mid\|x\|_{\mathbb{R}^{3}}<h_{0}\right\} \subset \mathbb{R}^{3} .
$$

The conditions (5) mean that, at time $t=0$, any two sets $S_{i}\left(z_{i}(0)\right)$ do not overlap, and that the swimmer lies in $\Omega$. The condition (6) is a regularity assumption of Lipschitz type regarding the shift of the set $S(0)$. It is satisfied, for instance, for balls and parallelepipeds.

Assumption 2. Assume that within some $\left(\mathbb{R}^{3}\right)^{n}$ neighborhood $\mathbf{G}(z(0)) \subset\left(\mathbb{R}^{3}\right)^{n}$ of the initial datum in (2) the mappings $A_{i}$ and $B_{i}$ are Lipschitz for all $i=$ $2, \ldots, n-1$ in the following sense:

$$
\begin{aligned}
& \left\|A_{i}\left(a_{i-1}-a_{i}\right)-A_{i}\left(b_{i-1}-b_{i}\right)\right\|_{\mathbb{R}^{3}} \\
& \leq L\left\{\left\|a_{i-1}-b_{i-1}\right\|_{\mathbb{R}^{3}}+\left\|a_{i}-b_{i}\right\|_{\mathbb{R}^{3}}\right. \\
& \left.\quad+\left\|a_{i+1}-b_{i+1}\right\|_{\mathbb{R}^{3}}\right\}, \\
& \left\|B_{i}\left(a_{i+1}-a_{i}\right)-B_{i}\left(b_{i+1}-b_{i}\right)\right\|_{\mathbb{R}^{3}} \\
& \leq L\left\{\left\|a_{i-1}-b_{i-1}\right\|_{\mathbb{R}^{3}}+\left\|a_{i}-b_{i}\right\|_{\mathbb{R}^{3}}\right. \\
& \left.\quad+\left\|a_{i+1}-b_{i+1}\right\|_{\mathbb{R}^{3}}\right\},
\end{aligned}
$$

for any $a_{i \pm 1}, a_{i}, b_{i \pm 1}, b_{i} \in \mathbf{G}(z(0))$, where $L>$ 0 is a constant. In the above, $A_{i}$ 's and $B_{i}$ 's are defined according to Section 1.2 by the respective triplets of vectors $\left\{a_{i-1}, a_{i}, a_{i+1}\right\}$ or $\left\{b_{i-1}, b_{i}, b_{i+1}\right\}$, i.e., depending on the vectors they act upon.

In particular, Assumption 2 holds if none of the triplets $\left\{z_{i-1}(0), z_{i}(0), z_{i+1}(0)\right\}, i=1, \ldots, n-1$, form a straight line, see Example 1. Here is the main result of this paper.

\section{Theorem 1. Let}

$$
\begin{gathered}
y_{0} \in H(\Omega), \quad T>0, k_{i}>0, \quad i=1, \ldots, n-1, \\
v_{i} \in L^{\infty}(0, T), \quad i=1, \ldots, n-2, \\
z_{i}(0) \in \Omega, \quad i=1, \ldots, n,
\end{gathered}
$$

and let Assumptions 1 and 2 hold. Then there exists $a T^{*}=T^{*}\left(z_{1}(0), \ldots, z_{n}(0),\left\|v_{1}\right\|_{L^{\infty}(0, T)}\right.$, $\left.\ldots,\left\|v_{n-2}\right\|_{L^{\infty}(0, T)}, \Omega\right) \in(0, T)$ such that the system (1)-(3) admits a unique solution $\{y, p, z\}$ on $\left(0, T^{*}\right)$, $\{y, \nabla p, z\} \in L^{2}\left(0, T^{*} ; J_{o}(\Omega)\right) \times L^{2}\left(0, T^{*} ; G(\Omega)\right) \times$ $\left[C\left(\left[0, T^{*}\right] ; \mathbb{R}^{3}\right)\right]^{n}$. Moreover, y $\in C\left(\left[0, T^{*}\right] ; H(\Omega)\right)$, $y_{t}, y_{x_{i} x_{j}}, p_{x_{i}} \in\left(L^{2}\left(Q_{T^{*}}\right)\right)^{3}$, where $i, j=1,2,3$, and Eqns. (1) and (2) are satisfied almost everywhere, while Assumptions 1 and 2 hold in $\left[0, T_{*}\right]$.

Remark 1. (Discussion of Theorem 1)

(i) It follows from the discussion at the end of Section 1.2 that Theorem 1 is primarily aimed at the well-posedness of the model (1)-(3) on the time-intervals between the "plane choice instants". This is due to the lack of continuity of mappings $\left\{A_{i}, B_{i}\right\}$, required by Assumption 2, at these instants. Despite that, let us point out that the parts of (3) dealing with the rotational forces can still be continuous in time at the "plane choice instants" if we select the respective scalar parameters $v_{i}(t)$ to be continuous and vanishing at these instants. This, in our opinion, opens up certain possibilities (which are not the subject of this paper) to study the well-posedness of (1)-(3) on the time-intervals which can include such instants.

(ii) The fact that the conditions Assumptions 1 and 2 hold in $\left[0, T_{*}\right]$ implies that we are able to guarantee that within $\left[0, T^{*}\right]$ no parts of the swimmer's body will "collide", and simultaneously, that it stays strictly inside of $\Omega$. These conditions allow us to maintain the mathematical and physical well-posedness of the model (1)-(3).

(iii) As will follow from the proof below, Theorem 1 allows further extension of the solutions to (1)-(3) in time as long as Assumptions 1 and 2 continue to hold, and the model stays between the "plane choice instants". This depends, in particular, on the choice of parameters $v_{1}(t), \ldots, v_{n-2}(t)$.

Our plan to prove Theorem 1 is to proceed stepwise as follows. In Section 3 we discuss the existence and uniqueness of the solutions to the decoupled version of (2). In Section 4 we will introduce three continuous 
mappings for the decoupled version of the system (1)-(3). In Section 5 we will apply a fixed-point argument to prove Theorem 1.

In the proofs below we employ the methods introduced by Khapalov and Eubanks (2009) to investigate the well-posedness of the 2-D version of the model (1)-(3), modifying and extending them to the 3-D case.

Without loss of generality, we will further assume that the system (1)-(3) and all respective auxiliary systems below are considered on the time-intervals whose lengths are smaller than 1 .

Throughout the paper we use the symbol $C$ to denote generic, possibly different, constants.

\section{Preliminary results}

Introduce the following decoupled version of the system (2):

$$
\begin{aligned}
& \frac{\mathrm{d} w_{i}}{\mathrm{~d} t}=\frac{1}{\operatorname{mes}\left(S_{i}(0)\right)} \int_{S_{i}\left(w_{i}(t)\right)} u(x, t) \mathrm{d} x, \\
& w_{i}(0)=z_{i, 0},
\end{aligned}
$$

where $i=1, \ldots, n$ and $u(x, t)$ is some given function. Write $w(t)=\left(w_{1}(t), \ldots, w_{n}(t)\right)$.

Lemma 1. Let $T>0$ and $u \in\left(L^{2}\left(0, T ; L^{\infty}(\Omega)\right)\right)^{3}$ be given. Then there is a $T^{*} \in(0, T)$ such that system (7) has a unique solution in $C\left(\left[0, T^{*}\right] ; \mathbb{R}^{3}\right)$ satisfying Assumptions 1 and 2 with $w(t)$ in place of $z(t)$, if they hold at time $t=0$.

Proof. We shall use the contraction principle to prove existence and uniqueness. Below, the values of $h_{0}, C$ are taken from (6).

Select $T_{0}$ to satisfy the following inequalities:

$$
\begin{aligned}
0< & T_{0} \\
< & \min \left\{\frac{\operatorname{mes}\left(S_{0}\right) h_{0}^{2}}{4\|u\|_{\left(L^{2}\left(Q_{T}\right)\right)^{3}}^{2}}, \frac{\left(\operatorname{mes}\left(S_{0}\right)\right)^{2}}{C^{2}\|u\|_{\left(L^{2}\left(0, T ; L^{\infty}(\Omega)\right)\right)^{3}}^{2}} T, 1\right\} \\
\leq & \min _{i=1, \ldots, n} \min \left\{\frac{\operatorname{mes}\left(S_{i}(0)\right) h_{0}^{2}}{4\|u\|_{\left(L^{2}\left(Q_{T}\right)\right)^{3}}^{2}},\right. \\
& \left.\frac{\left(\operatorname{mes}\left(S_{i}(0)\right)\right)^{2}}{C^{2}\|u\|_{\left(L^{2}\left(0, T ; L^{\infty}(\Omega)\right)\right)^{3}}^{2}} T, 1\right\} .
\end{aligned}
$$

Let, for any given $p \in C\left(\left[0, T_{0}\right] ; \mathbb{R}^{3}\right)$,

$$
\begin{aligned}
& C\left(\left[0, T_{0}\right] ; \mathbb{R}^{3}\right) \supseteq B_{h_{0} / 2}(p) \\
& =\left\{z \in C\left(\left[0, T_{0}\right] ; \mathbb{R}^{3}\right) \mid\|z-p\|_{C\left(\left[0, T_{0}\right] ; \mathbb{R}^{3}\right)} \leq \frac{h_{0}}{2}\right\} .
\end{aligned}
$$

For each $i=1, \ldots, n$, define a mapping $D_{i}$ : $B_{h_{0} / 2}\left(z_{i, 0}\right) \longrightarrow C\left(\left[0, T_{0}\right] ; \mathbb{R}^{3}\right)$ by

$$
\begin{aligned}
& D_{i}\left(w_{i}(t)\right) \\
& \quad=z_{i, 0}+\frac{1}{\operatorname{mes}\left(S_{i}(0)\right)} \int_{0}^{t} \int_{S_{i}\left(w_{i}(\tau)\right)} u(x, \tau) \mathrm{d} x \mathrm{~d} \tau .
\end{aligned}
$$

Then we can derive that, for $t \in\left[0, T_{0}\right], i=1, \ldots, n$,

$$
\begin{aligned}
& \left\|D_{i}\left(w_{i}(t)\right)\right\|_{\mathbb{R}^{3}} \\
& \quad \leq\left\|z_{i, 0}\right\|_{\mathbb{R}^{3}}+\frac{\sqrt{T_{0}}}{\sqrt{\operatorname{mes}\left(S_{i}(0)\right)}}\|u\|_{\left(L^{2}\left(Q_{T}\right)\right)^{3}} .
\end{aligned}
$$

Similarly, in view of (8),

$$
\begin{aligned}
& \left\|D_{i}\left(w_{i}(t)\right)-z_{i, 0}\right\|_{C\left(\left[0, T_{0}\right] ; \mathbb{R}^{3}\right)} \\
& \quad \leq \frac{\sqrt{T_{0}}}{\sqrt{\operatorname{mes}\left(S_{i}(0)\right)}}\|u\|_{\left(L^{2}\left(Q_{T}\right)\right)^{3}}<\frac{h_{0}}{2} .
\end{aligned}
$$

Thus, $D_{i}$ maps $B_{h_{0} / 2}\left(z_{i, 0}\right)$ into itself for each $i=$ $1, \ldots, n$, where $z_{i, 0}$ is treated as a constant function.

Let $w_{i}^{(1)}(t), w_{i}^{(2)}(t) \in B_{h_{0} / 2}\left(z_{i, 0}\right)$ and $\xi(x, S)$ denote the characteristic function of a set $S \subset \mathbb{R}^{3}$. Then, making use of (6), we obtain

$$
\begin{aligned}
&\left\|D_{i}\left(w_{i}^{(1)}(t)\right)-D_{i}\left(w_{i}^{(2)}(t)\right)\right\|_{\mathbb{R}^{3}}^{2} \\
&=\frac{1}{\operatorname{mes}^{2}\left(S_{i}(0)\right)} \| \int_{0}^{t} \int_{S_{i}\left(w_{i}^{(1)}(t)\right)} u(x, \tau) \mathrm{d} x \mathrm{~d} \tau \\
&-\int_{0}^{t} \int_{S_{i}\left(w_{i}^{(2)}(\tau)\right)} u(x, \tau) \mathrm{d} x \mathrm{~d} \tau \|_{\mathbb{R}^{3}}^{2} \\
&= \frac{1}{\operatorname{mes}^{2}\left(S_{i}(0)\right)} \| \int_{0}^{t} \int_{\Omega} u(x, \tau)\left(\xi\left(x, S_{i}\left(w_{i}^{(1)}(t)\right)\right)\right. \\
&\left.-\xi\left(x, S_{i}\left(w_{i}^{(2)}(\tau)\right)\right)\right) \mathrm{d} x \mathrm{~d} \tau \|_{\mathbb{R}^{3}}^{2} \\
& \leq \frac{1}{\operatorname{mes}^{2}\left(S_{i}(0)\right)} \sum_{j=1}^{3}\left[\int_{0}^{t} \int_{\Omega}\left|u_{j}(x, \tau)\right|\right. \\
&\left.\times\left|\xi\left(x, S_{i}\left(w_{i}^{(1)}(t)\right)\right)-\xi\left(x, S_{i}\left(w_{i}^{(2)}(\tau)\right)\right)\right| \mathrm{d} x \mathrm{~d} \tau\right]^{2} \\
& \leq \frac{1}{\operatorname{mes}^{2}\left(S_{i}(0)\right)} \sum_{j=1}^{3}\left[\int_{0}^{t}\|u(\cdot, \tau)\|_{\left(L^{\infty}(\Omega)\right)^{3}}\right. \\
&\left.\times \int_{S_{i}\left(w_{i}^{(1)}(t)\right) \Delta S_{i}\left(w_{i}^{(2)}(\tau)\right)} 1 \mathrm{~d} x \mathrm{~d} \tau\right]^{2},
\end{aligned}
$$

where we set $u(x, \tau)=\left(u_{1}(x, \tau), u_{2}(x, \tau), u_{3}(x, \tau)\right)$ and 
used the fact, cf. (6), that for $i=1, \ldots, n$

$$
\begin{aligned}
\mid & \xi\left(x, S_{i}\left(w_{i}^{(1)}(t)\right)\right)-\xi\left(x, S_{i}\left(w_{i}^{(2)}(\tau)\right)\right) \mid \\
=\mid & \xi\left(x, S_{i}\left(w_{i}^{(1)}(t)\right)\right) \\
& -\xi\left(x, S_{i}\left(w_{i}^{(1)}(t)-\left(w_{i}^{(1)}(t)-w_{i}^{(2)}(\tau)\right)\right) \mid\right. \\
= & \begin{cases}1, & \text { if } x \in S_{i}\left(w_{i}^{(1)}(t)\right) \Delta S_{i}\left(w_{i}^{(2)}(\tau)\right), \\
0, & \text { if } x \text { is elsewhere in } \Omega .\end{cases}
\end{aligned}
$$

We will apply next (6) with

$$
h=w_{i}^{(1)}(t)-w_{i}^{(2)}(t), \quad x=w_{i}^{(1)}(t),
$$

and, after that, we will employ Hölder's inequality with respect to the integration over $t$ :

$$
\begin{aligned}
&\left\|D_{i}\left(w_{i}^{(1)}(t)\right)-D_{i}\left(w_{i}^{(2)}(t)\right)\right\|_{\mathbb{R}^{3}}^{2} \\
& \leq \frac{C^{2}}{\operatorname{mes}^{2}\left(S_{i}(0)\right)}\left\|w_{i}^{(1)}-w_{i}^{(2)}\right\|_{C\left(\left[0, T_{0}\right] ; \mathbb{R}^{3}\right)}^{2} \\
& \quad \times \sum_{j=1}^{3}\left[\int_{0}^{t}\|u(\cdot, \tau)\|_{\left(L^{\infty}(\Omega)\right)^{3}} \mathrm{~d} \tau\right]^{2} \\
& \leq \frac{C^{2} t}{\operatorname{mes}^{2}\left(S_{i}(0)\right)}\left\|w_{i}^{(1)}-w_{i}^{(2)}\right\|_{C\left(\left[0, T_{0}\right] ; \mathbb{R}^{3}\right)}^{2} \\
& \quad \times \sum_{j=1}^{3}\left[\int_{0}^{t}\|u(\cdot, \tau)\|_{\left(L^{\infty}(\Omega)\right)^{3}}^{2} \mathrm{~d} \tau\right] \\
& \leq \frac{3 C^{2} T_{0}}{\operatorname{mes}^{2}\left(S_{i}(0)\right)} \\
& \times\left\|w_{i}^{(1)}-w_{i}^{(2)}\right\|_{C\left(\left[0, T_{0}\right] ; \mathbb{R}^{3}\right)}^{2}\|u\|_{\left(L^{2}\left(0, T_{0} ; L^{\infty}(\Omega)\right)\right)^{3}}^{2} .
\end{aligned}
$$

Finally, replacing $3 C^{2}$ with a generic notation $C^{2}$ for a constant (see the remark before Section 3 ) and taking the square root, we arrive at, for $t \in\left[0, T_{0}\right], i=1, \ldots, n$,

$$
\begin{aligned}
& \left\|D_{i}\left(w_{i}^{(1)}(t)\right)-D_{i}\left(w_{i}^{(2)}(t)\right)\right\|_{\mathbb{R}^{3}} \\
& \leq \frac{C \sqrt{T_{0}}}{\operatorname{mes}\left(S_{i}(0)\right)}\|u\|_{\left(L^{2}\left(0, T ; L^{\infty}(\Omega)\right)\right)^{3}} \\
& \quad \times\left\|w_{i}^{(1)}-w_{i}^{(2)}\right\|_{C\left(\left[0, T_{0}\right] ; \mathbb{R}^{3}\right)} .
\end{aligned}
$$

Therefore, after maximizing the left-hand side of (10) over $\left[0, T_{0}\right]$, we conclude that

$$
\begin{aligned}
& \left\|D_{i}\left(w_{i}^{(1)}(t)\right)-D_{i}\left(w_{i}^{(2)}(t)\right)\right\|_{C\left(\left[0, T_{0}\right] ; \mathbb{R}^{3}\right)} \\
& \leq \frac{C \sqrt{T_{0}}}{\operatorname{mes}\left(S_{i}(0)\right)}\|u\|_{\left(L^{2}(0, T ; L \infty(\Omega))\right)^{3}} \\
& \quad \times\left\|w_{i}^{(1)}-w_{i}^{(2)}\right\|_{C\left(\left[0, T_{0}\right] ; \mathbb{R}^{3}\right)} .
\end{aligned}
$$

Hence, in view of (8),

$$
\frac{C \sqrt{T_{0}}}{\operatorname{mes}\left(S_{i}(0)\right)}\|u\|_{\left(L^{2}\left(0, T ; L^{\infty}(\Omega)\right)\right)^{3}}<1,
$$

it follows from (11) that $D_{i}$ is a contraction mapping on $B_{h_{0} / 2}\left(z_{i, 0}\right)$ for each $i=1, \ldots, n$. Therefore, there exist unique $w_{i}(t) \in C\left(\left[0, T_{0}\right] ; \mathbb{R}^{3}\right), i=1, \ldots, n$, such that $D_{i}\left(w_{i}(t)\right)=w_{i}(t)$, i.e., for $i=1, \ldots, n$,

$$
\begin{aligned}
w_{i}(t) & =z_{i, 0} \\
+ & \frac{1}{\operatorname{mes}\left(S_{i}(0)\right)} \int_{0}^{t} \int_{S_{i}\left(w_{i}(\tau)\right)} u(x, \tau) \mathrm{d} x \mathrm{~d} \tau,
\end{aligned}
$$

which yields (7).

The estimates (9a-b) imply that we may select a $T^{*} \in\left(0, T_{0}\right)$ such that for any $t \in\left[0, T^{*}\right]$, all $w_{i}(t)$ 's will stay "close enough" to their initial values $z_{i, 0}$ to guarantee that Assumptions 1 and 2 hold for $w_{i}(t), i=1, \ldots, n$. This ends the proof of Lemma 1.

\section{Decoupled solution mappings}

Let $\mathcal{B}_{q}(0)$ denote a closed ball of radius $q$ (its value will be selected in Section 5) with center at the origin in the Banach space $L^{2}\left(0, T ; J_{o}(\Omega)\right) \cap L^{2}\left(0, T ;\left(H^{2}(\Omega)\right)^{3}\right)$ endowed with the norm of $L^{2}\left(0, T ;\left(H^{2}(\Omega)\right)^{3}\right)$,

$$
\begin{gathered}
\mathcal{B}_{q}(0)=\left\{\phi \in L^{2}\left(0, T ; J_{o}(\Omega)\right) \cap L^{2}\left(0, T ;\left(H^{2}(\Omega)\right)^{3}\right) \mid\right. \\
\left.\|\phi\|_{L^{2}\left(0, T ;\left(H^{2}(\Omega)\right)^{3}\right)} \leq q\right\},
\end{gathered}
$$

where $H^{2}(\Omega)=\left\{\phi \mid \phi, \phi_{x_{i}}, \phi_{x_{i} x_{j}} \in L^{2}(\Omega), i, j=\right.$ $1,2,3\}$.

Note that $H^{2}(\Omega)$ is continuously embedded into $C(\bar{\Omega})$, and thus $L^{2}\left(0, T ;\left(H^{2}(\Omega)\right)^{3}\right)$ is continuously embedded into $\left(L^{2}\left(0, T ; L^{\infty}(\Omega)\right)\right)^{3}$. This yields the estimate

$$
\|\phi\|_{\left(L^{2}\left(0, T ; L^{\infty}(\Omega)\right)\right)^{3}} \leq K\|\phi\|_{L^{2}\left(0, T ;\left(H^{2}(\Omega)\right)^{3}\right)}
$$

for some $K>0$. This implies that Lemma 1 holds for any $u \in \mathcal{B}_{q}(0)$.

4.1. Solution mapping for $z_{i}(t), i=1, \ldots, n$. We now intend to show that the operator

$$
\begin{gathered}
\mathbf{A}: \mathcal{B}_{q}(0) \rightarrow\left[C\left([0, T] ; \mathbb{R}^{3}\right)\right]^{n}, \\
\mathbf{A} u=w=\left(w_{1}, \ldots, w_{n}\right),
\end{gathered}
$$

where the $w_{i}$ 's solve (7), is continuous and compact if $T>0$ is sufficiently small.

Continuity. Let $u^{(1)}, u^{(2)} \in \mathcal{B}_{q}(0)$ with $T_{1}$ in place of $T$, where $T_{1}>0$ satisfies assumptions in the proof of Lemma 1 with $T_{1}$ in place of $T^{*}$. Define $\mathbf{A} u^{(j)}=$ $w^{(j)}=\left(w_{1}^{(j)}, \ldots, w_{n}^{(j)}\right)$ for $j=1,2$. To show that $\mathbf{A}$ is continuous, we shall evaluate

$$
\left\|\mathbf{A} u^{(1)}-\mathbf{A} u^{(2)}\right\|_{\left[C\left([0, T] ; \mathbb{R}^{3}\right)\right]^{n}}
$$


term-by-term. To this end, similarly to (10), we have the following estimate:

$$
\begin{aligned}
& \left\|w_{i}^{(1)}(t)-w_{i}^{(2)}(t)\right\|_{\mathbb{R}^{3}} \\
& =\| \frac{1}{\operatorname{mes}\left(S_{i}(0)\right)} \int_{0}^{t} \int_{S\left(w_{i}^{(1)}(\tau)\right)} u^{(1)}(x, \tau) \mathrm{d} x \mathrm{~d} \tau \\
& -\frac{1}{\operatorname{mes}\left(S_{i}(0)\right)} \int_{0}^{t} \int_{S\left(w_{i}^{(2)}(\tau)\right)} u^{(2)}(x, \tau) \mathrm{d} x \mathrm{~d} \tau \|_{\mathbb{R}^{3}} \\
& =\frac{1}{\operatorname{mes}\left(S_{i}(0)\right)} \| \int_{0}^{t} \int_{S\left(w_{i}^{(1)}(\tau)\right)} u^{(1)}(x, \tau) \mathrm{d} x \mathrm{~d} \tau \\
& -\int_{0}^{t} \int_{S\left(w_{i}^{(1)}(\tau)\right)} u^{(2)}(x, \tau) \mathrm{d} x \mathrm{~d} \tau \\
& +\int_{0}^{t} \int_{S\left(w_{i}^{(1)}(\tau)\right)} u^{(2)}(x, \tau) \mathrm{d} x \mathrm{~d} \tau \\
& -\int_{0}^{t} \int_{S\left(w_{i}^{(2)}(\tau)\right)} u^{(2)}(x, \tau) \mathrm{d} x \mathrm{~d} \tau \|_{\mathbb{R}^{3}} \\
& \leq \frac{1}{\operatorname{mes}\left(S_{i}(0)\right)}\left(\| \int_{0}^{t} \int_{\Omega}\left(u^{(1)}(x, \tau)-u^{(2)}(x, \tau)\right)\right. \\
& \times \xi\left(x, S\left(w_{i}^{(1)}(\tau)\right)\right) \mathrm{d} x \mathrm{~d} \tau\left\|_{\mathbb{R}^{3}}+\right\| \int_{0}^{t} \int_{\Omega} u^{(2)}(x, \tau) \\
& \left.\times\left(\xi\left(x, S\left(w_{i}^{(1)}(\tau)\right)\right)-\xi\left(x, S\left(w_{i}^{(2)}(\tau)\right)\right)\right) \mathrm{d} x \mathrm{~d} \tau \|_{\mathbb{R}^{3}}\right) \\
& \leq \frac{\sqrt{T_{1}}}{\sqrt{\operatorname{mes}\left(S_{0}\right)}}\left\|u^{(1)}-u^{(2)}\right\|_{\left(L^{2}\left(Q_{T_{1}}\right)\right)^{3}} \\
& +\frac{C \sqrt{T_{1}}}{\operatorname{mes}\left(S_{0}\right)}\left\|u^{(2)}\right\|_{\left(L^{2}\left(0, T_{1} ; L^{\infty}(\Omega)\right)\right)^{3}} \\
& \times\left\|w_{i}^{(1)}-w_{i}^{(2)}\right\|_{C\left(\left[0, T_{1}\right] ; \mathbb{R}^{3}\right.} .
\end{aligned}
$$

Recall from (13) that

$$
\left\|u^{(2)}\right\|_{\left(L^{2}\left(0, T_{1} ; L^{\infty}(\Omega)\right)\right)^{3}} \leq K q .
$$

Therefore, select a $T>0$ satisfying

$$
0<T<\min \left\{\left(\frac{\operatorname{mes}\left(S_{0}\right)}{C K q}\right)^{2}, T_{1}\right\} .
$$

Hence, replacing $T_{1}$ in (14) with $T$ satisfying (15) and maximizing the left-hand side of (14) over $[0, T]$, we obtain

$$
\begin{aligned}
& \left\|w_{i}^{(1)}-w_{i}^{(2)}\right\|_{C\left([0, T] ; \mathbb{R}^{2}\right)} \\
& \leq \frac{\sqrt{T}}{\sqrt{\operatorname{mes}\left(S_{0}\right)}}\left\|u^{(1)}-u^{(2)}\right\|_{\left(L^{2}\left(Q_{T}\right)\right)^{3}} \\
& \quad+\frac{C K q \sqrt{T}}{\operatorname{mes}\left(S_{0}\right)}\left\|w_{i}^{(1)}-w_{i}^{(2)}\right\|_{C\left([0, T] ; \mathbb{R}^{3}\right)} .
\end{aligned}
$$

In view of $(15)$, if $w_{i}^{(1)}(t) \neq w_{i}^{(2)}(t)$ on $[0, T]$, then the above implies

$$
\begin{aligned}
0 & <\left(1-\frac{C K q \sqrt{T}}{\operatorname{mes}\left(S_{0}\right)}\right)\left\|w_{i}^{(1)}-w_{i}^{(2)}\right\|_{C\left([0, T] ; \mathbb{R}^{3}\right)} \\
& \leq \frac{\sqrt{T}}{\sqrt{\operatorname{mes}\left(S_{0}\right)}}\left\|u^{(1)}-u^{(2)}\right\|_{\left(L^{2}\left(Q_{T}\right)\right)^{3}} .
\end{aligned}
$$

Thus, it follows that

$$
\begin{aligned}
& \left\|w_{i}^{(1)}-w_{i}^{(2)}\right\|_{C\left([0, T] ; \mathbb{R}^{3}\right)} \\
& \leq \frac{\sqrt{T \operatorname{mes}\left(S_{0}\right)}}{\operatorname{mes}\left(S_{0}\right)-C K q \sqrt{T}}\left\|u^{(1)}-u^{(2)}\right\|_{\left(L^{2}\left(Q_{T}\right)\right)^{3}} .
\end{aligned}
$$

Therefore, (15) and (16) imply that, for every $u^{(1)}$, $u^{(2)} \in \mathcal{B}_{q}(0)$,

$$
\begin{aligned}
& \left\|\mathbf{A} u^{(1)}-\mathbf{A} u^{(2)}\right\|_{\left[C\left([0, T] ; \mathbb{R}^{3}\right)\right]^{n}} \\
& \leq \frac{\sqrt{n T \operatorname{mes}\left(S_{0}\right)}}{\operatorname{mes}\left(S_{0}\right)-C K q \sqrt{T}}\left\|u^{(1)}-u^{(2)}\right\|_{L^{2}\left(0, T ;\left(H^{2}(\Omega)\right)^{3}\right)} .
\end{aligned}
$$

The operator $\mathbf{A}$ is continuous on $\mathcal{B}_{q}(0)$ for sufficiently small $T$ as in (15).

Compactness. Furthermore, to show that $\mathbf{A}$ is compact, we will show that $\mathbf{A}$ maps any sequence in $\mathcal{B}_{q}(0)$ into a sequence in $\left[C\left([0, T] ; \mathbb{R}^{3}\right)\right]^{n}$ which contains a convergent subsequence. To this end, consider any sequence $\left\{u^{(j)}\right\}_{j=1}^{\infty}$ in $\mathcal{B}_{q}(0)$. Using (12) with $w_{i}^{(j)}$ and $u^{(j)}$ in place of $w_{i}$ and $u$, construct the sequence $\left\{w_{i}^{(j)}\right\}_{j=1}^{\infty}, i=1, \ldots, n$.

Let us now show that $\left\{w_{i}^{(j)}\right\}_{j=1}^{\infty}$ is uniformly bounded and equicontinuous. Indeed, applying (13) to an estimate like (9a) and then maximizing over $[0, T]$ yield

$$
\begin{aligned}
& \left\|w_{i}^{(j)}\right\|_{C\left([0, T] ; \mathbb{R}^{3}\right)} \\
& \quad \leq \max _{i=1, \ldots, n}\left\{\left\|z_{i, 0}\right\|_{\mathbb{R}^{3}}\right\}+\frac{q \sqrt{T}}{\sqrt{\operatorname{mes}\left(S_{0}\right)}} .
\end{aligned}
$$

To show equicontinuity, consider any $t, t+h \in$ $[0, T]$, e.g., when $h>0$. Then for $i=1, \ldots, n, j=$ $1, \ldots$,

$$
\begin{aligned}
& \left\|w_{i}^{(j)}(t+h)-w_{i}^{(j)}(t)\right\|_{\mathbb{R}^{3}} \\
& =\frac{1}{\operatorname{mes}\left(S_{i}(0)\right)}\left\|\int_{t}^{t+h} \int_{S\left(w_{i}^{(j)}(\tau)\right)} u^{(j)}(x, \tau) \mathrm{d} x \mathrm{~d} \tau\right\|_{\mathbb{R}^{3}} \\
& \leq \frac{\sqrt{h}}{\sqrt{\operatorname{mes}\left(S_{i}(0)\right)}}\left\|u^{(j)}\right\|_{\left(L^{2}\left(Q_{T}\right)\right)^{3}} \leq \frac{q \sqrt{h}}{\sqrt{\operatorname{mes}\left(S_{0}\right)}}
\end{aligned}
$$


which implies the equicontinuity of $\left\{w_{i}^{(j)}\right\}_{j=1}^{\infty}, i=$ $1, \ldots, n$ (the case $h<0$ is similar). Therefore, by Ascoli's theorem, $\left\{\mathbf{A} u^{(j)}\right\}_{j=1}^{\infty}$ contains a convergent subsequence in $\left[C\left([0, T] ; \mathbb{R}^{3}\right)\right]^{n}$, i.e., $\mathbf{A}$ is compact on $\mathcal{B}_{q}(0)$.

4.2. Solution mapping for decoupled Stokes equations. Now, consider the following decoupled Stokes initial boundary value problem:

$$
\frac{\partial y_{*}}{\partial t}-\nu \Delta y_{*}+\nabla p_{*}=f(x, t) \text { in } Q_{T}
$$

$\operatorname{div} y_{*}=0$ in $Q_{T}, \quad y_{*}=0 \quad$ in $\Sigma_{T},\left.y_{*}\right|_{t=0}=y_{0} \in H(\Omega)$.

For any $f \in\left(L^{2}\left(Q_{T}\right)\right)^{3}$, it is known that the boundary value problem (18) possesses a unique solution $y_{*}$ in $L^{2}\left(0, T ; J_{o}(\Omega)\right) \cap L^{2}\left(0, T ;\left(H^{2}(\Omega)\right)^{3}\right)$, with the properties described in Theorem 1 (see, e.g., Ladyzhenskaya, 1963; Temam, 1984). Moreover (see, e.g., Ladyzhenskaya, 1963, (7), (50)), there is a positive constant $L$ such that

$$
\begin{aligned}
& \left\|y_{*}\right\|_{L^{2}\left(0, T ;\left(H^{2}(\Omega)\right)^{3}\right)}^{2} \\
& \quad \leq L\left\|y_{0}\right\|_{H(\Omega)}^{2}+L \int_{Q_{T}}\|f(\cdot, \tau)\|_{\mathbb{R}^{3}}^{2} \mathrm{~d} \tau .
\end{aligned}
$$

Thus it follows that, given $y_{0}$, the operator

B :

$\left(L^{2}\left(Q_{T}\right)\right)^{3} \rightarrow L^{2}\left(0, T ; J_{o}(\Omega)\right) \cap L^{2}\left(0, T ;\left(H^{2}(\Omega)\right)^{3}\right)$,

$\mathbf{B} f=y_{*}$, is continuous.

4.3. Term $\boldsymbol{F}(\boldsymbol{z}, \boldsymbol{v})$. Let $T>0$ be as in (15). Given $u \in \mathcal{B}_{q}(0)$, let $F_{*}(w), w=\left(w_{1}, \ldots, w_{n}\right)$ denote the value of $F(z, v)$ in (3), with $w_{i}$ 's from (12) in $z_{i}$ 's, respectively. Consider the operator

$$
\mathbf{F}:\left[C\left([0, T] ; \mathbb{R}^{3}\right)\right]^{n} \longrightarrow\left(L^{2}\left(Q_{T}\right)\right)^{3}, \quad \mathbf{F} w=F_{*}(w) .
$$

We will show that $\mathbf{F}$ is continuous, but first we evaluate

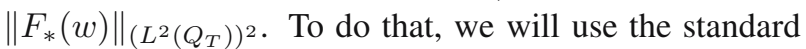
algebraic transformation technique similar to that used for the 2-D swimming model by Khapalov and Eubanks (2009) as well as Khapalov (2010). Therefore, we omit some of the details below.

Let $P(T)$ denote the upper bound in (17):

$$
P(T)=\max _{i=1, \ldots, n}\left\{\left\|z_{i, 0}\right\|_{\mathbb{R}^{3}}\right\}+\frac{q \sqrt{T}}{\sqrt{\operatorname{mes}\left(S_{0}\right)}} .
$$

Then, using (17) with $w_{i}$ in place of $w_{i}^{(j)}$, we can evaluate the first term in square brackets in the first line of (3) as follows:

$$
\begin{aligned}
& \| k_{i-1} \frac{\left(\left\|w_{i}(t)-w_{i-1}(t)\right\|_{\mathbb{R}^{3}}-l_{i-1}\right)}{\left\|w_{i}(t)-w_{i-1}(t)\right\|_{\mathbb{R}^{3}}} \\
& \quad \times\left(w_{i}(t)-w_{i-1}(t)\right) \|_{\mathbb{R}^{3}} \\
& \quad \leq C_{0}\left(n, r, P(T), \max _{i=1, \ldots, n-1}\left\{k_{i}\right\}, \max _{i=1, \ldots n-1}\left\{l_{i}\right\}\right),
\end{aligned}
$$

where

$$
C_{0}=C_{0}\left(n, r, P(T), \max _{i=1, \ldots, n-1}\left\{k_{i}\right\}, \max _{i=1, \ldots n-1}\left\{l_{i}\right\}\right)
$$

denotes a positive generic constant, continuous in $r, k_{i}$ 's, $l_{i}$ 's, and $P(T)$ (it can have a different concrete value for different expressions below). Along similar estimates for other terms in (3), we obtain, for $u \in \mathcal{B}_{q}(0)$,

$$
\begin{aligned}
& \left\|F_{*}(w)\right\|_{\left(L^{2}\left(Q_{T}\right)\right)^{3}} \\
& \leq C_{o} \sqrt{T \operatorname{mes}(\Omega)}\left(1+\max _{i=1, \ldots, n-2}\left\{\left\|v_{i}\right\|_{L^{\infty}(0, T)}\right\}\right) .
\end{aligned}
$$

We will now show that $\mathbf{F}$ is a continuous operator Let $w^{(1)}, w^{(2)} \in\left[C\left([0, T] ; \mathbb{R}^{3}\right)\right]^{n}$. Consider (3) with $w_{i}^{(j)}$ in place of $z_{i}, i=1, \ldots, n, j=1,2$.

Making use of an estimate like (9b), without loss of generality we can assume that $T$ is small enough to ensure that Assumptions 1 and 2 hold (as stated in Lemma 1). Then, once again, similar to the 2-D case model of Khapalov and Eubanks (2009), we can evaluate $\left\|F_{*}\left(w^{(1)}\right)-F_{*}\left(w^{(2)}\right)\right\|_{\left(L^{2}\left(Q_{T}\right)\right)^{3}}$ along the standard algebraic transformations and making use of Assumption 2 (instead of matrix $A$ of Khapalov and Eubanks (2009) used in the 2-D as described in Section 1.2).

Namely, the terms in the first two lines in (3) are the same as the respective terms in the work of Khapalov and Eubanks (2009), except that we will deal now with the 3-D vectors instead of 2-D. The main difference is the use of nonlinear transformations $A_{i}$ and $B_{i}$ instead of the $2 \times 2$ matrix $A$ of Khapalov and Eubanks (2009). Therefore, we will need to employ the estimate in Assumption 2. For example, consider

$$
\begin{aligned}
& \| v_{i-1}(t) \xi_{i-1}(x, t)\left(A_{i}\left(w_{i-1}^{(1)}(t)-w_{i}^{(1)}(t)\right)\right) \\
& -v_{i-1}(t) \xi_{i-1}(x, t)\left(A_{i}\left(w_{i-1}^{(2)}(t)-w_{i}^{(2)}(t)\right)\right) \|_{\left(L^{2}(\Omega)\right)^{3}} \\
& \leq \sqrt{\operatorname{mes}(\Omega)}\left\|v_{i-1}(t)\right\|_{L^{\infty}(0, T)} \\
& \quad \times \|\left(A_{i}\left(w_{i-1}^{(1)}(t)-w_{i}^{(1)}(t)\right)\right)
\end{aligned}
$$




$$
\begin{aligned}
& -\left(A_{i}\left(w_{i-1}^{(2)}(t)-w_{i}^{(2)}(t)\right)\right) \|_{\mathbb{R}^{3}} \\
\leq & \sqrt{\operatorname{mes}(\Omega)}\left\|v_{i-1}(t)\right\|_{L^{\infty}(0, T)} \\
& \times L\left\{\left\|w_{i-1}^{(1)}(t)-w_{i-1}^{(2)}(t)\right\|_{\mathbb{R}^{3}}\right. \\
& +\left\|w_{i}^{(1)}(t)-w_{i}^{(2)}(t)\right\|_{\mathbb{R}^{3}} \\
& \left.+\left\|w_{i+1}^{(1)}(t)-w_{i+1}^{(2)}(t)\right\|_{\mathbb{R}^{3}}\right\} \\
\leq & 3 L\left\|w^{(1)}-w^{(2)}\right\|_{\left[C\left([0, T] ; \mathbb{R}^{3}\right)\right]^{n}},
\end{aligned}
$$

which proves the continuity of the first term in the third line in (3) with respect to the $\left[C\left([0, T] ; \mathbb{R}^{3}\right)\right]^{n}$ norm. Of course, $A_{i}$ can be replaced with $B_{i}$ in the above type of calculations. In turn, the second term in line 3 in (3), is the product of two continuous functions (the scalar and 3-D). The continuity of the terms in the last line of (3) follows from the above argument.

The above calculations will result in the following formula:

$$
\begin{aligned}
& \left\|F_{*}\left(w^{(1)}\right)-F_{*}\left(w^{(2)}\right)\right\|_{\left(L^{2}\left(Q_{T}\right)\right)^{3}} \\
\leq & M(T) \sqrt{T \operatorname{mes}(\Omega)}\left\|w^{(1)}-w^{(2)}\right\|_{\left[C\left([0, T] ; \mathbb{R}^{3}\right)\right]^{n}},
\end{aligned}
$$

where

$$
\begin{aligned}
& M(T) \\
& =M\left(T, n, r, P(T), \max _{i=1, \ldots, n-1}\left\{k_{i}\right\}, \max _{i=1, \ldots n-1}\left\{l_{i}\right\}\right) \\
& \quad \times\left(1+\max _{j=1, \ldots, n-2}\left\{\left\|v_{j}\right\|_{L^{\infty}(0, T)}\right\}\right), \quad
\end{aligned}
$$

is defined similarly to $C_{o}$. Hence $\mathbf{F}$ is a continuous operator for $T$ satisfying (15).

\section{Proof of Theorem 1}

Before proceeding to the proof of Theorem 1, we summarize the main results of the previous section. In Section 4, we proved that, for sufficiently small $T>0$ satisfying (4.3), the operators

$$
\begin{gathered}
\mathbf{A}: \mathcal{B}_{q}(0) \rightarrow\left[C\left([0, T] ; \mathbb{R}^{3}\right)\right]^{n}, \\
\mathbf{A} u=w=\left(w_{1}, \ldots, w_{n}\right), \\
\mathbf{F}:\left[C\left([0, T] ; \mathbb{R}^{3}\right)\right]^{n} \longrightarrow\left(L^{2}\left(Q_{T}\right)\right)^{3}, \quad \mathbf{F} w=F_{*}(w),
\end{gathered}
$$

and

B :

$$
\left(L^{2}\left(Q_{T}\right)\right)^{3} \longrightarrow L^{3}\left(0, T ; J_{o}(\Omega)\right) \cap L^{2}\left(0, T ;\left(H^{2}(\Omega)\right)^{3}\right),
$$

$\mathbf{B} f=y_{*}$, are all continuous. A is also compact. As a result,

BFA :

$$
B_{q}(0) \rightarrow L^{2}\left(0, T ; J_{o}(\Omega)\right) \cap L^{2}\left(0, T ;\left(H^{2}(\Omega)\right)^{3}\right),
$$

$\mathbf{B F A} u=y_{*}$, is continuous and compact.
5.1. Existence: Fixed point argument. Select the value of $q$ to be any positive number larger than $\sqrt{L}\left\|y_{0}\right\|_{H(\Omega)}$, and choose $T>0$ as in (15) so that Lemma 1 holds. In view of (19) (with $F_{*}$ in place of $f$ ), we select $T^{*} \in(0, \min \{T, 1\})$ small enough so that the continuous and compact operator BFA maps the closed ball $B_{q}(0)$ into itself.

Rewrite (21) as follows:

$$
\left\|F_{*}(w)\right\|_{\left(L^{2}\left(Q_{T}\right)\right)^{3}}<C_{1} \sqrt{T},
$$

where the positive constant $C_{1}$ is independent of $T$. Select $T^{*}$ so that

$$
0<T^{*}<\min \left\{\frac{q^{2}-L\left\|y_{0}\right\|_{H(\Omega)}^{2}}{L C_{1}^{2}}, T, 1\right\} .
$$

Since $T^{*}$ satisfies (15), if we replace $T$ with $T^{*}$ in (19), (21)-(23), we obtain

$$
\begin{aligned}
& \| \text { BFA } u \|_{L^{2}\left(0, T^{*} ;\left(H^{2}(\Omega)\right)^{3}\right)}^{2} \\
& \quad \leq L\left\|y_{0}\right\|_{H(\Omega)}^{2}+L\left\|F_{*}(w)\right\|_{\left(L^{2}\left(Q_{T^{*}}\right)\right)^{3}}^{2} \\
& \quad<L\left\|y_{0}\right\|_{H(\Omega)}^{2}+L C_{1}^{2} T^{*} \\
& \quad<L\left\|y_{0}\right\|_{H(\Omega)}^{2}+q^{2}-L\left\|y_{0}\right\|_{H(\Omega)}^{2}=q^{2} .
\end{aligned}
$$

Hence, BFA maps $\mathcal{B}_{q}(0)$ into itself if (24) is satisfied.

Thus, by Schauder's fixed point theorem, BFA has a fixed point $y$ which is a solution of the system (1)-(3), and satisfies all of the requirements of Theorem 1. As usual, we may select $\nabla p$ in $L^{2}\left(0, T^{*} ; G(\Omega)\right)$ to complement the solution $y \in L^{2}\left(0, T^{*} ; J_{o}(\Omega)\right)$ in Theorem 1. This completes the proof of existence for Theorem 1.

5.2. Uniqueness. To prove that the solution found in Section 5.1 is unique, we will argue by contradiction. Namely, suppose, e.g., that there are two different solutions

$$
\left\{z^{(1)}=\left(z_{1}^{(1)}, \ldots, z_{n}^{(1)}\right), y^{(1)}, p^{(1)}\right\}
$$

and

$$
\left\{z^{(2)}=\left(z_{1}^{(2)}, \ldots, z_{n}^{(2)}\right), y^{(2)}, p^{(2)}\right\}
$$

to (1)-(3), satisfying the properties described in Theorem 1 on some time interval $[0, T]$, where $T$ satisfies the inequality (24). Without loss of generality, we assume that the two solutions are different right from $t=0$.

By (16), with $z_{i}^{(j)}$ in place of $w_{i}^{(j)}, i=1, \ldots, n$, and $y^{(j)}$ in place of $u^{(j)}$, both for $j=1,2$, we see that, for any $T_{0} \in(0, T]$ and each $i=1, \ldots, n$,

$$
\begin{aligned}
& \left\|z_{i}^{(1)}-z_{i}^{(2)}\right\|_{C\left(\left[0, T_{0}\right] ; \mathbb{R}^{3}\right)} \\
& \leq \frac{\sqrt{T_{0} \operatorname{mes}\left(S_{0}\right)}}{\operatorname{mes}\left(S_{0}\right)-C K q \sqrt{T_{0}}}\left\|y^{(1)}-y^{(2)}\right\|_{\left(L^{2}\left(Q_{T_{0}}\right)\right)^{3} .}
\end{aligned}
$$


Let us now evaluate $\left\|y^{(1)}-y^{(2)}\right\|_{\left(L^{2}\left(Q_{T_{0}}\right)\right)^{3}}$. Note that $\left(y^{(1)}-y^{(2)}\right)$ satisfies the following Stokes initial-value problem:

$$
\begin{gathered}
\frac{\partial\left(y^{(1)}-y^{(2)}\right)}{\partial t}= \\
\nu \Delta\left(y^{(1)}-y^{(2)}\right) \\
+\left(F\left(z^{(1)}, v\right)-F\left(z^{(2)}, v\right)\right) \\
-\nabla\left(p^{(1)}-p^{(2)}\right) \text { in } Q_{T_{0}}, \\
\operatorname{div}\left(y^{(1)}-y^{(2)}\right)=0 \quad \text { in } Q_{T_{0}}, \\
\left(y^{(1)}-y^{(2)}\right)=0 \quad \text { in } \Sigma_{T_{0}}, \\
\left.\left(y^{(1)}-y^{(2)}\right)\right|_{t=0}=0 . \\
\text { According to }(19) \text { we have } \\
\left\|y^{(1)}-y^{(2)}\right\|_{\left(L^{2}\left(Q_{T_{0}}\right)\right)^{3}}^{2} \\
\leq L \int_{0}^{T_{0}} \int_{\Omega}\left\|F\left(z^{(1)}, v\right)-F\left(z^{(2)}, v\right)\right\|_{\mathbb{R}^{3}}^{2} \mathrm{~d} x \mathrm{~d} t .
\end{gathered}
$$

In turn, similarly to (22)

$$
\begin{aligned}
& \| F\left(z^{(1)}, v\right)-F\left(z^{(2)}, v\right) \|_{\mathbb{R}^{3}} \\
& \leq N(T) \sum_{j=1}^{n}\left\|z_{j}^{(1)}-z_{j}^{(2)}\right\|_{C\left(\left[0, T_{0}\right] ; \mathbb{R}^{3}\right)} .
\end{aligned}
$$

for some $N(T)$ is nonincreasing at $T \rightarrow 0^{+}$. Hence, combining (24)-(26) yields

$$
\begin{aligned}
& \left\|y^{(1)}-y^{(2)}\right\|_{\left(L^{2}\left(Q_{T_{0}}\right)\right)^{3}} \\
\leq & \frac{n N(T) T_{0} \sqrt{L \operatorname{mes}\left(S_{0}\right) \operatorname{mes}(\Omega)}}{\operatorname{mes}(S(0))-C K q \sqrt{T_{0}}}\left\|y^{(1)}-y^{(2)}\right\|_{\left(L^{2}\left(Q_{T_{0}}\right)\right)^{3}} .
\end{aligned}
$$

Now, select $T_{0}$ as follows:

$$
\begin{aligned}
0< & T_{0} \\
< & \min \left\{\frac{\operatorname{mes}\left(S_{0}\right)}{4 n N(T) \sqrt{L \operatorname{mes}\left(S_{0}\right) \operatorname{mes}(\Omega)}},\right. \\
& \left.\left(\frac{\operatorname{mes}\left(S_{0}\right)}{2 C K q}\right)^{2}, T\right\} .
\end{aligned}
$$

This choice of $T_{0}$ implies that the following inequality holds:

$n N(T) T_{0} \sqrt{L \operatorname{mes}\left(S_{0}\right) \operatorname{mes}(\Omega)}+\frac{1}{2} C k q \sqrt{T_{0}}<\frac{1}{2} \operatorname{mes}\left(S_{0}\right)$.

Hence from (27) and (28), it follows that

$$
\left\|y^{(1)}-y^{(2)}\right\|_{\left(L^{2}\left(Q_{T_{0}}\right)\right)^{3}}<\frac{1}{2}\left\|y^{(1)}-y^{(2)}\right\|_{\left(L^{2}\left(Q_{T_{0}}\right)\right)^{3}} .
$$

Therefore $y^{(1)} \equiv y^{(2)}$ on $\left[0, T_{0}\right]$, and thus, by (25), $z_{i}^{(1)} \equiv$ $z_{i}^{(2)}$ for $i=1, \ldots, n$ on $\left[0, T_{0}\right]$, a contradiction. This ends the proof of Theorem 1 .

\section{Acknowledgment}

This work was supported in part by the NSF grant DMS-1007981.

\section{References}

Alouges, F., DeSimone, A. and Lefebvre, A. (2008). Optimal Strokes for low Reynolds number swimmers: An example, Journal of Nonlinear Science 18: 27-302.

Becker, L.E, Koehler, S.A. and Stone, H.A. (2003). On self-propulsion of micro-machines at low Reynolds number: Purcell's three-link swimmer, Journal of Fluid Mechanics 490: 15-35.

Belter, D. and Skrzypczyński, P (2010). A biologically inspired approach to feasible gait learning for a hexapod robot, International Journal of Applied Mathematics and Computer Science 20(1): 69-84, DOI: 10.2478/v10006-010-0005-7.

Childress, S. (1981). Mechanics of Swimming and Flying, Cambridge University Press, Cambridge.

Dal Maso, Gi., DeSimone, A. and Morandotti, M. (2011) An existence and uniqueness result for the motion of self-propelled micro-swimmers, SIAM Journal of Mathematical Analysis 43: 1345-1368.

Fukuda, T., Kawamoto, A., Arai, F. and Matsuura, H. (1995). Steering mechanism and swimming experiment of micro mobile robot in water, Proceedings of Micro Electro Mechanical Systems (MEMS'95), Amsterdam, The Netherlands, pp. 300-305.

Fauci, L. and Peskin, C.S. (1988). A computational model of aquatic animal locomotion, Journal of Computational Physics 77: 85-108.

Fauci, L. (1993). Computational modeling of the swimming of biflagellated algal cells, Contemporary Mathematics 141: 91-102.

Galdi, G.P. (1999). On the steady self-propelled motion of a body in a viscous incompressible fluid, Archive for Rational Mechanics and Analysis 1448: 53-88.

Galdi, G.P. (2002). On the motion of a rigid body in a viscous liquid: A mathematical analysis with applications, in $\mathrm{S}$. Friedlander and D. Serre (Eds.), Handbook of Mathematical Fluid Mechanics, Elsevier Science, Amsterdam, pp. 653-791.

Gray, J. (1932). Study in animal locomotion IV: The propulsive power of the dolphin, Journal of Experimental Biology 10: 192-199.

Gray, J. and Hancock, G.J. (1955). The propulsion of sea-urchin spermatozoa, Journal of Experimental Biology 32: 802.

Guo, S. (2002). Afin type of micro-robot in pipe, Proceedings of the 2002 International Symposium on Micromechatronics and Human Science (MHS 2002), Ngoya, Japan, pp. 93-98.

Gurtin, M.E. (1981). An Introduction to Continuum Mechanics, Academic Press, New York, NY. 
Hawthorne, M.F., Zink, J.I., Skelton, J.M., Bayer, M.J., Liu, Ch., Livshits, E., Baer, R. and Neuhauser, D. (2004). Electrical or photocontrol of rotary motion of a metallacarborane, Science 303: 1849.

Happel, V. and Brenner, H. (1965). Low Reynolds Number Hydrodynamics, Prentice Hall, Upper Saddle River, NJ.

Hirose, S. (1993). Biologically Inspired Robots: Snake-like Locomotors and Manipulators, Oxford University Press, Oxford.

Kanso. E., Marsden, J.E., Rowley, C.W. and Melli-Huber, J. (2005). Locomotion of articulated bodies in a perfect fluid, Journal of Nonlinear Science 15: 255-289.

Khapalov, A.Y. (1999). Approximate controllability properties of the semilinear heat equation with lumped controls, International Journal of Applied Mathematics and Computer Science 9(4): 751-765.

Khapalov, A.Y. (2005). The well-posedness of a model of an apparatus swimming in the 2-D Stokes fluid, Technical Report 2005-5, Washington State University, Department of Mathematics, http://www.math.wsu.edu/TRS/2005-5.pdf

Khapalov, A.Y. and Eubanks, S. (2009). The wellposedness of a 2-D swimming model governed in the nonstationary Stokes fluid by multiplicative controls, Applicable Analysis 88: 1763-1783, DOI:10.1080/00036810903401222.

Khapalov, A.Y. (2010). Controllability of Partial Differential Equations Governed by Multiplicative Controls, Lecture Notes in Mathematics Series, Vol. 1995, Springer-Verlag, Berlin/Heidelberg.

Khapalov, A.Y. and Trinh, G. (2013). Geometric aspects of transformations of forces acting upon a swimmer in a 3-D incompressible fluid, Discrete and Continuous Dynamical Systems Series A 33: 1513-1544.

Khapalov, A.Y. (2013). Micro motions of a swimmer in the 3-D incompressible fluid governed by the nonstationary Stokes equation, arXiv: 1205.1088 [math.AP], (preprint).

Koiller, J., Ehlers, F. and Montgomery, R. (1996). Problems and progress in microswimming, Journal of Nonlinear Science 6: $507-541$.

Ladyzhenskaya, O.A. (1963). The Mathematical Theory of Viscous Incompressible Flow, Cordon and Breach, New York, NY.

Lighthill, M.J. (1975). Mathematics of Biofluid Dynamics, Society for Industrial and Applied Mathematics, Philadelphia, PA.

Mason, R. and Burdick, J.W. (2000). Experiments in carangiform robotic fish locomotion, Proceedings of the IEEE International Conference on Robotics and Automation, San Francisco, CA, USA, pp. 428-435.
McIsaac, K.A. and Ostrowski, J.P. (2000). Motion planning for dynamic eel-like robots, Proceedings of the IEEE International Conference on Robotics and Automation, San Francisco, CA, USA, pp. 1695-1700.

Martinez, S. and Cortes, J. (2001). Geometric control of robotic locomotion systems, Proceedings of the 10th Fall Workshop on Geometry and Physics, Miraflores de la Sierra, Spain, Vol. 4, pp. 183-198.

Morgansen, K.A., Duindam, V., Mason, R.J., Burdick, J.W. and Murray, R.M. (2001). Nonlinear control methods for planar carangiform robot fish locomotion, Proceedings of the IEEE International Conference on Robotics and Automation, Seoul, Korea, pp. 427-434.

Peskin, C.S. (1977). Numerical analysis of blood flow in the heart, Journal of Computational Physics 25: 220-252.

Peskin, C.S. and McQueen, D.M. (1994). A general method for the computer simulation of biological systems interacting with fluids, SEB Symposium on Biological Fluid Dynamics, Leeds, UK.

San Martin, J., Takashi, T. and Tucsnak, M. (2007). A control theoretic approach to the swimming of microscopic organisms, Quarterly Applied Mathematics 65: 405-424.

San Martin, J., Scheid, J.-F., Takashi, T. and Tucsnak, M. (2008). An initial and boundary value problem modeling of fish-like swimming, Archive of Rational Mechanics and Analysis 188: 429-455.

Shapere, A. and Wilczeck, F. (1989). Geometry of self-propulsion at low Reynolds number, Journal of Fluid Mechanics 198: 557-585.

Sigalotti, M. and Vivalda, J.-C. (2009). Controllability properties of a class of systems modeling swimming microscopic organisms, ESAIM: Control, Optimisation and Calculus of Variations 16: 1053-1076.

Taylor, G.I. (1951). Analysis of the swimming of microscopic organisms, Proceeding of the Royal Society of London A 209: 447-461.

Taylor, G.I. (1952). Analysis of the swimming of long and narrow animals, Proceedings of the Royal Society of London A 214(1117): 158-183.

Temam, R. (1984). Navier-Stokes Equations, North-Holland, Amsterdam.

Trintafyllou, M.S., Trintafyllou, G.S. and Yue, D.K.P. (2000). Hydrodynamics of fishlike swimming, Annual Review of Fluid Mechanics 32: 33-53.

Tytell, E.D., Hsu, C.-Y, Williams, T.L., Cohen, A.H. and Fauci, L.J. (2010). Interactions between internal forces, body stiffness, and fluid environment in a neuromechanical model of lamprey swimming, Proceedings of the National Academy Sciences, USA 107: 19832-19837.

Wu, T.Y. (1971). Hydrodynamics of swimming fish and cetaceans, Advances in Applied Mathematics 11: 1-63. 


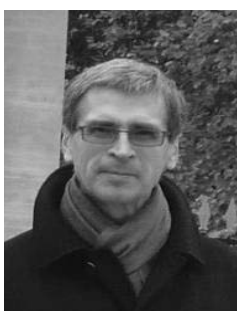

Alexander Y. Khapalov received his Ph.D. in mathematics from the Institute of Mathematics and Mechanics, Yekaterinburg, Russia (USSR) in 1982. Currently, he is a full professor in the Department of Mathematics, Washington State University, USA. His present research is focused on multiplicative controllability theory for PDEs with applications to parabolic and hyperbolic equations and to swimming models. He has recently published a research monograph entitled Controllability of Partial Differential Equations Governed by Multiplicative Controls (Springer-Verlag) on this topic.

Received: 19 April 2012

Revised: 18 October 2012

Re-revised: 14 January 2013 